\title{
Equivalence of analytic and rational functions
}

\author{
by J. Bochnak (Amsterdam), M. Buchner (Albuquerque, N. Mex.) \\ and W. Kucharz (Albuquerque, N. Mex.)
}

\begin{abstract}
We give a criterion for a real-analytic function defined on a compact nonsingular real algebraic set to be analytically equivalent to a rational function.

Throughout this paper $M$ denotes a compact nonsingular algebraic subset of $\mathbb{R}^{n}$. As usual, by a polynomial function on $M$ we mean the restriction to $M$ of a polynomial function from $\mathbb{R}^{n}$ into $\mathbb{R}$. A function $r: A \rightarrow \mathbb{R}$, defined on a subset $A$ of $M$, is said to be regular on $A$ if there exist polynomial functions $p: M \rightarrow \mathbb{R}$ and $q: M \rightarrow \mathbb{R}$ such that $q^{-1}(0) \cap A=\emptyset$ and $r(x)=p(x) / q(x)$ for all $x$ in $A$ (in other words, $r$ is regular on $A$ if it is a rational function on $M$, whose denominator is nonzero at each point of $A$ ). Of course, every polynomial function is regular, and every regular function on $M$ is (real-) analytic. Two analytic functions $f: M \rightarrow \mathbb{R}$ and $g: M \rightarrow \mathbb{R}$ are called analytically equivalent if $g=f \circ \sigma$ for some analytic diffeomorphism $\sigma: M \rightarrow M$. In the present paper we are concerned with the following problem: What conditions have to be imposed on an analytic function $f: M \rightarrow \mathbb{R}$ in order for it to be analytically equivalent to a polynomial or a regular function on $M$ ?

The reader may consult $[1,2,5,6]$ for earlier results related to this problem. Let us consider now an analytic function $f: M \rightarrow \mathbb{R}$ with isolated critical points. It is known that $f$ is not necessarily analytically equivalent to a regular function on $M[1, \mathrm{pp} .416,417]$, and therefore one has to impose some extra conditions (let us recall, however, that for each nonnegative
\end{abstract}

1991 Mathematics Subject Classification: 32B15, 32S20.

Key words and phrases: analytic function, rational function, analytic equivalence.

The first author supported in part by CEE contract ERBCHRXCT 940506.

The second author supported in part by a RAC grant at the University of New Mexico.

The third author supported in part by an NSF grant and a RAC grant at the University of New Mexico.

All authors were supported in part by NATO Collaborative Research Grants Programme, CRG 930238. 
integer $k$ one can find a $C^{k}$ diffeomorphism $\sigma: M \rightarrow M$ such that $f \circ \sigma$ is a polynomial function on $M\left[2\right.$, Theorem 8.2]). Denote by $M_{\mathbb{C}}$ the smallest complex algebraic subset of $\mathbb{C}^{n}$ containing $M$ (we view $\mathbb{R}^{n}$ as a subset of $\mathbb{C}^{n}$ ). Obviously, every point of $M$ is a nonsingular point of $M_{\mathbb{C}}$. Furthermore, every analytic function $g: M \rightarrow \mathbb{R}$ has a unique extension $g_{\mathbb{C}}:\left(M_{\mathbb{C}}, M\right) \rightarrow \mathbb{C}$ to a holomorphic function-germ at $M$. If each critical point of $f$ is an isolated critical point of the holomorphic function-germ $f_{\mathbb{C}}:\left(M_{\mathbb{C}}, M\right) \rightarrow \mathbb{C}$, then $f$ is analytically equivalent to a polynomial function on $M[1$, Theorem 5].

We always consider $M$ endowed with the usual metric topology induced from $\mathbb{R}^{n}$. Given a point $x$ in $M$ and a function $g: U \rightarrow \mathbb{R}$ defined in a neighborhood of $x$, we denote by $g_{x}$ the germ of $g$ at $x$. Two analytic functiongerms $\varphi:(M, x) \rightarrow \mathbb{R}$ and $\psi:(M, x) \rightarrow \mathbb{R}$ are said to be analytically equivalent if there exists a local analytic diffeomorphism $\tau:(M, x) \rightarrow(M, x)$ such that $\psi=\varphi \circ \tau$. If $h: M \rightarrow \mathbb{R}$ is a differentiable function, then $\Sigma_{h}$ will denote the set of critical points of $h$.

Conjecture 1. Let $f: M \rightarrow \mathbb{R}$ be an analytic function with isolated critical points. Assume that for each point $x$ in $\Sigma_{f}$ the germ $f_{x}$ is analytically equivalent to the germ at $x$ of a regular function defined in a neighborhood of $x$. Then $f$ is analytically equivalent to a regular function on $M$.

We shall prove a somewhat weaker result than Conjecture 1, but first we need some preparation. Denote by $\mathcal{E}_{x}$ the ring of all $C^{\infty}$ function-germs $(M, x) \rightarrow \mathbb{R}$ at a point $x$ in $M$. Given $\varphi$ in $\mathcal{E}_{x}$, we define $\triangle(\varphi)$ to be the ideal of $\mathcal{E}_{x}$ generated by

$$
\frac{\partial \varphi}{\partial x_{1}}, \ldots, \frac{\partial \varphi}{\partial x_{m}}
$$

where $m=\operatorname{dim} M$ and $\left(x_{1}, \ldots, x_{m}\right)$ is a local $C^{\infty}$ coordinate system in a neighborhood of $x$ in $M$. Assume that $x$ is a critical point of $\varphi$, that is, $\triangle(\varphi) \neq \mathcal{E}_{x}$. Although we shall not use it later on, let us observe, in order to motivate the definition given below, that the following conditions are equivalent:

(a) $x$ is an isolated critical point of $\varphi$

(b) there exists $\lambda$ in $\triangle(\varphi)$ such that $\lambda^{-1}(0)=\{0\}$ as set-germs;

(c) there exists a $C^{\infty}$ function $u: M \rightarrow \mathbb{R}$ such that $u^{-1}(0)=\{x\}$ and the germ $u_{x}$ belongs to $\triangle(\varphi)$.

Indeed, if (a) is satisfied, then so is (b) with $\lambda=\left(\frac{\partial \varphi}{\partial x_{1}}\right)^{2}+\ldots+\left(\frac{\partial \varphi}{\partial x_{m}}\right)^{2}$. If (b) holds, then, using partition of unity, one easily constructs a $C^{\infty}$ function $u: M \rightarrow \mathbb{R}$ such that $u^{-1}(0)=\{x\}$ and $u_{x}=\lambda^{2}$, which implies (c). It is clear that (a) follows from (c).

Furthermore, if $\varphi$ is an analytic function-germ, then, applying the theory of coherent real analytic sheaves, one can prove that (a), (b), (c) are equivalent to 
$\left(\mathrm{c}^{\prime}\right)$ there exists an analytic function $v: M \rightarrow \mathbb{R}$ such that $v^{-1}(0)=\{x\}$ and the germ $v_{x}$ belongs to $\triangle(\varphi)$.

Definition 2. We say that the critical point $x$ of $\varphi$ is algebraically isolated in $M$ if there exists a regular (or, equivalently, a polynomial) function $r: M \rightarrow \mathbb{R}$ such that $r^{-1}(0)=\{x\}$ and the germ $r_{x}$ belongs to $\triangle(\varphi)$.

Denote by $\mathcal{E}(M)$ the ring of all $C^{\infty}$ functions on $M$. Let $X_{1}, \ldots, X_{d}$ be $C^{\infty}$ vector fields on $M$ generating the $\mathcal{E}(M)$-module of all $C^{\infty}$ vector fields on $M$. Given a $C^{\infty}$ function $f: M \rightarrow \mathbb{R}$, we denote by $\triangle(f)$ the ideal of $\mathcal{E}(M)$ generated by $X_{1} f, \ldots, X_{d} f$; clearly, $\triangle(f)$ does not depend on the choice of the generators $X_{1}, \ldots, X_{d}$. By construction, for each point $x$ in $M$, we have $\triangle(f) \mathcal{E}_{x}=\triangle\left(f_{x}\right)$.

We shall need the following fact.

EXAmPle 3. Let $g: M \rightarrow \mathbb{R}$ be a regular function on $M$. Assume that $\Sigma_{g} \cap g^{-1}(g(x))=\{x\}$ for some point $x$ in $M$. We claim that $x$ is a critical point of $g_{x}$ algebraically isolated in $M$. In order to prove the claim, we choose polynomial vector fields $X_{1}, \ldots, X_{d}$ on $M$ generating the $\mathcal{E}(M)$-module of all $C^{\infty}$ vector fields on $M$, and set

$$
r=\left(X_{1} g\right)^{2}+\ldots+\left(X_{d} g\right)^{2}+(g-g(x))^{2 m},
$$

where $m=\operatorname{dim} M$ (a vector field $X$ on $M$ is said to be a polynomial vector field if $X p$ is a polynomial function on $M$ for every polynomial function $p: M \rightarrow \mathbb{R})$. Obviously, $r$ is a regular function on $M$. Since $\Sigma_{g}$ is equal to the set of zeros of $s=\left(X_{1} g\right)^{2}+\ldots+\left(X_{d} g\right)^{2}$ and since $\Sigma_{g} \cap g^{-1}(g(x))=\{x\}$, we obtain $r^{-1}(0)=\{x\}$. It is clear that $s$ belongs to $\triangle(g)$. By [3], the germ $\left(g_{x}-g(x)\right)^{m}$ is in $\triangle\left(g_{x}\right)$, and therefore $r_{x}$ belongs to $\triangle\left(g_{x}\right)$. Thus the claim is proved.

Recall that a local $C^{1}$ diffeomorphism $\tau:(M, x) \rightarrow(M, x)$ is said to be orientation preserving if $\operatorname{det}\left(D_{x} \tau\right)>0$, where $D_{x} \tau: T_{x} M \rightarrow T_{x} M$ is the derivative of $\tau$ at $x$.

ExAmple 4 . Let $\varphi:(M, x) \rightarrow \mathbb{R}$ be an analytic function-germ and let $\varphi_{\mathbb{C}}:\left(M_{\mathbb{C}}, x\right) \rightarrow \mathbb{C}$ be the unique extension of $\varphi$ to a holomorphic functiongerm. Assume that $x$ is an isolated critical point of $\varphi_{\mathbb{C}}$. It is well known that there exists a local orientation preserving analytic diffeomorphism $\tau$ : $(M, x) \rightarrow(M, x)$ such that $\varphi \circ \tau$ is the germ at $x$ of a polynomial function on $M$ [7, p. 170, Proposition 4.2, p. 59, Théorème 4.2].

We assert that $x$ is a critical point of both $\varphi$ and $\varphi \circ \tau$ algebraically isolated in $M$. Of course, it suffices to prove the assertion for $\varphi$. Denote by $m\left(\mathcal{E}_{x}\right)$ the unique maximal ideal of $\mathcal{E}_{x}$; obviously,

$$
m\left(\mathcal{E}_{x}\right)=\left\{\lambda \in \mathcal{E}_{x} \mid \lambda(x)=0\right\} .
$$


Since $x$ is an isolated critical point of $\varphi_{\mathbb{C}}$, it follows that some power of $m\left(\mathcal{E}_{x}\right)$, say $m\left(\mathcal{E}_{x}\right)^{l}$, is contained in $\triangle(\varphi)[7$, p. 170 , Proposition 4.2 and its proof]. Choose a regular function $s: M \rightarrow \mathbb{R}$ such that $s^{-1}(0)=\{x\}$ and set $r=s^{l}$. Then $r^{-1}(0)=\{x\}$ and $r_{x}$ belongs to $\triangle(\varphi)$, which implies our assertion.

THEOREM 5. Let $f: M \rightarrow \mathbb{R}$ be an analytic function with isolated critical points. Assume that for each point $x$ in $\Sigma_{f}$, there exists a local orientation preserving analytic diffeomorphism $\sigma_{x}:(M, x) \rightarrow(M, x)$ such that $f_{x} \circ \sigma_{x}$ is the germ at $x$ of a regular function defined in a neighborhood of $x$, and $x$ is a critical point of $f_{x} \circ \sigma_{x}$ algebraically isolated in $M$. Then $f$ is analytically equivalent to a regular function on $M$.

For the proof of Theorem 5 we shall need the following.

LEMMA 6. Let $x$ be a point in $M$ and let $\psi:(M, x) \rightarrow \mathbb{R}$ be the germ at $x$ of a regular function defined in a neighborhood of $x$. Assume that $x$ is a critical point of $\psi$ algebraically isolated in $M$. Then there exist a regular function $g: M \rightarrow \mathbb{R}$ and a local orientation preserving analytic diffeomorphism $\tau:(M, x) \rightarrow(M, x)$ such that $\Sigma_{g} \cap g^{-1}(g(x))=\{x\}$ and $\psi \circ \tau=g_{x}$.

Proof. Let $r: M \rightarrow \mathbb{R}$ be a regular function such that $r^{-1}(0)=\{x\}$ and $r_{x}$ belongs to $\triangle(\psi)$. Pick polynomial functions $p: M \rightarrow \mathbb{R}$ and $q: M \rightarrow \mathbb{R}$ such that $q(x) \neq 0$ and $\psi=p_{x} / q_{x}$. Note that the function

$$
u=\frac{p q+r^{4}}{q^{2}+r^{4}}
$$

is regular on $M$.

An obvious modification of [2, Lemma 3.2] implies the existence of a $C^{\infty}$ function $f: M \rightarrow \mathbb{R}$ such that $\Sigma_{f} \cap f^{-1}(f(x))=\{x\}, f_{x}=\psi$, and $f \mid M \backslash\{x\}$ has only nondegenerate critical points. Note that

$$
f_{x}-u_{x}=\psi-u_{x}=r_{x}^{4} \frac{p_{x}-q_{x}}{q_{x}\left(q_{x}^{2}+r_{x}^{4}\right)} .
$$

Applying this and the equality $r^{-1}(0)=\{x\}$, we can find a $C^{\infty}$ function $\alpha: M \rightarrow \mathbb{R}$ satisfying

$$
f=u+r^{4} \alpha
$$

Let $\mathcal{V}$ be a neighborhood of 0 in the $C^{\infty}$ topology on $\mathcal{E}(M)$ and let $\beta: M \rightarrow$ $\mathbb{R}$ be a polynomial function such that $\beta-\alpha$ is in $\mathcal{V}$ and $j_{y}^{1}(\beta)=j_{y}^{1}(\alpha)$ for all $y$ in $\Sigma_{f} \backslash\{x\}$, where $j_{y}^{1}(-)$ stands for the 1 -jet at $y$ ( $\beta$ exists in view of $[1$, Corollary 1]). Then

$$
g=u+r^{4} \beta
$$

is a regular function on $M$. 
By construction, $r_{x}^{2}$ belongs to $\triangle(f)^{2} \mathcal{E}_{x}=\triangle(\psi)^{2}$. Furthermore, for each $y$ in $\Sigma_{f} \backslash\{x\}$, we have $\triangle(f) \mathcal{E}_{y}=m\left(\mathcal{E}_{y}\right)$, and hence $\beta_{y}-\alpha_{y}$ belongs to $\triangle(f)^{2} \mathcal{E}_{y}$. Since $\Sigma_{f}$ is precisely the set of zeros of the ideal $\triangle(f)$, using (1), $(2)$, and partition of unity, we get

$$
g-f=r^{4}(\beta-\alpha) \in r^{2} \triangle(f)^{2} .
$$

It also follows from the observations recorded in this paragraph and from [7, p. 119, Corollaire 1.6] that $\triangle(f)^{2}$ is a closed ideal (in the $C^{\infty}$ topology) of $\mathcal{E}(M)$. Taking $\mathcal{V}$ sufficiently small and applying [2, Theorem 2.1] (with $G=\{1\}=$ the trivial subgroup of $\mathbb{R} \backslash\{0\})$, we obtain a $C^{\infty}$ diffeomorphism $\sigma: M \rightarrow M$ isotopic to the identity and such that $g=f \circ \sigma, \sigma(x)=x$. Hence $\Sigma_{g} \cap g^{-1}(g(x))=\{x\}$ and $g_{x}=f_{x} \circ \sigma_{x}=\psi \circ \sigma_{x}$. So, by construction, $\sigma_{x}:(M, x) \rightarrow(M, x)$ is a local orientation preserving $C^{\infty}$ diffeomorphism. By [7, p. 59, Théorème 4.2], there also exists a local analytic diffeomorphism $\tau:(M, x) \rightarrow(M, x)$ such that $g_{x}=\psi \circ \tau$ and $j_{x}^{1}(\tau)=j_{x}^{1}\left(\sigma_{x}\right)$. It follows from the last equality that $\tau$ is orientation preserving. Thus the lemma is proved.

Proof of Theorem 5. Let $\Sigma_{f}=\left\{x_{1}, \ldots, x_{k}\right\}$. By Lemma 6, for each $i=1, \ldots, k$ there exist a regular function $g_{i}: M \rightarrow \mathbb{R}$ and a local orientation preserving analytic diffeomorphism $\tau_{i}:\left(M, x_{i}\right) \rightarrow\left(M, x_{i}\right)$ such that $\Sigma_{g_{i}} \cap g_{i}^{-1}\left(g_{i}\left(x_{i}\right)\right)=\left\{x_{i}\right\}$ and $f_{x_{i}} \circ \tau_{i}=g_{i x_{i}}$. Let $\sigma: M \rightarrow M$ be a $C^{\infty}$ diffeomorphism satisfying $\sigma_{x_{i}}=\tau_{i}$ for all $i=1, \ldots, k$. Then $f \circ \sigma=g_{i}$ in a neighborhood of $x_{i}$.

Choose a regular function $r_{i}: M \rightarrow \mathbb{R}$ such that $r_{i}^{-1}(0)=\left\{x_{i}\right\}$ and $r_{i x_{i}}$ belongs to $\triangle\left(g_{i x_{i}}\right)$ (cf. Example 3 ). Then $s=r_{1}^{2} \ldots r_{k}^{2}$ is a regular function on $M, s^{-1}(0)=\Sigma_{f}=\Sigma_{f \circ \sigma}$, and $s$ belongs to $\triangle(f \circ \sigma)^{2}$ (the last property follows by applying partition of unity). Note that

$$
u=\left(\sum_{i=1}^{k}\left(\prod_{j \neq i} r_{j}^{2}\right) g_{i}\right) /\left(\sum_{i=1}^{k}\left(\prod_{j \neq i} r_{j}^{2}\right)\right)
$$

is a regular function on $M$ and

$$
(f \circ \sigma)_{x_{l}}-u_{x_{l}}=g_{l x_{l}}-u_{x_{l}}=s_{x_{l}} v_{l},
$$

where $v_{l}$ is the germ at $x_{l}$ of the regular function

$$
\left(\sum_{i=1}^{k}\left(\prod_{\substack{j \neq i \\ j \neq l}} r_{j}^{2}\right)\left(g_{l}-g_{i}\right)\right) /\left(\left(\sum_{i=1}^{k} \prod_{j \neq i} r_{j}^{2}\right)\left(\prod_{j \neq l} r_{j}^{2}\right)\right)
$$

on $\left(M \backslash\left\{x_{1}, \ldots, x_{k}\right\}\right) \cup\left\{x_{l}\right\}$. It follows that we can find a $C^{\infty}$ function $\alpha: M \rightarrow \mathbb{R}$ satisfying $f \circ \sigma=u+s \alpha$. Let $\beta: M \rightarrow \mathbb{R}$ be a regular function 
and let $g=u+s \beta$. Then

$$
g-f \circ \tau=(\beta-\alpha) s \in \triangle(f \circ \sigma)^{2} .
$$

Let $\sigma^{*}: \mathcal{E}(M) \rightarrow \mathcal{E}(M)$ be the ring isomorphism defined by $\sigma^{*}(h)=h \circ \sigma$ for $h$ in $\mathcal{E}(M)$. Clearly, $\triangle(f \circ \sigma)^{2}=\sigma^{*}\left(\triangle(f)^{2}\right)$, and therefore, in view of [7, p. 119, Corollaire 1.6], the ideal $\triangle(f \circ \sigma)^{2}$ of $\mathcal{E}(M)$ is closed in the $C^{\infty}$ topology on $\mathcal{E}(M)$. By [2, Theorem 2.1], if $\beta$ is sufficiently close to $\alpha$ in the $C^{\infty}$ topology, then there exists a $C^{\infty}$ diffeomorphism $\eta: M \rightarrow M$ such that $g=f \circ \sigma \circ \eta$. It follows from the last equality and [4, Theorem 8.4] that $f$ and $g$ are analytically equivalent. Since $g$ is a regular function on $M$, the proof is complete.

\section{References}

[1] J. Bochnak, W. Kucharz and M. Shiota, On equivalence of ideals of real global analytic functions and the 17th Hilbert problem, Invent. Math. 63 (1981), 403-421.

[2] - - , - On algebraicity of global real analytic sets and functions, ibid. 70 (1982), $115-156$.

[3] J. Briançon et H. Skoda, Sur la clôture intégrale d'un idéal de germes de fonctions holomorphes en un point de $\mathbf{C}^{n}$, C. R. Acad. Sci. Paris Sér. A 278 (1974), 949-951.

[4] M. Shiota, Equivalence of differentiable mappings and analytic mappings, Inst. Hautes Études Sci. Publ. Math. 54 (1981), 237-322.

[5] —, Equivalence of differentiable functions, rational functions and polynomials, Ann. Inst. Fourier (Grenoble) 32 (1982), 167-204.

[6] R. Thom, L'équivalence d'une fonction différentiable et d'un polynôme, Topology 3 (1965), suppl. 2, 297-307.

[7] J. C. Tougeron, Idéaux de fonctions différentiables, Ergeb. Math. Grenzgeb. 71, Springer, 1972.

Department of Mathematics

Vrije Universiteit

De Boelelaan 1081a

1081 HV Amsterdam

The Netherlands

E-mail: bochnak@cs.vu.nl
Department of Mathematics and Statistics University of New Mexico Albuquerque, New Mexico 87131 U.S.A.

E-mail: buchner@math.unm.edu kucharz@math.unm.edu 